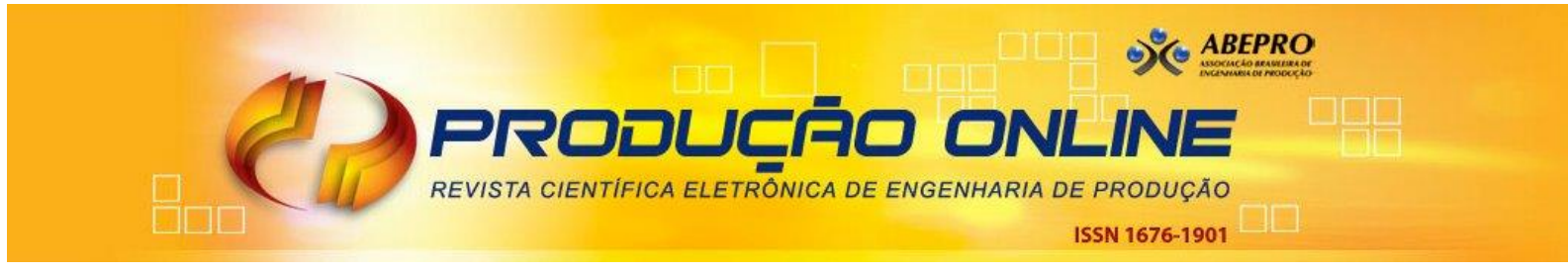

\title{
ANÁLISE MULTIVARIADA DE COMPONENTES PRINCIPAIS E MODELO MULTICRITÉRIO APLICADOS À DECISÃO DE LOCALIZAÇÃO DE EMPRESA
}

\section{MULTIVARIATE ANALYSIS OF PRINCIPAL COMPONENT AND MULTICRITERY MODEL APPLIED TO THE DECISION TO LOCATE A COMPANY}

\author{
Régis Façanha Dantas* E-mail: regis.dantas@uol.com.br \\ *Caixa Econômica Federal Governo do Estado do Ceará (CEF Ceará), Fortaleza, CE
}

Resumo: Dentre os fatores de sucesso de uma empresa, a escolha da localização é preponderante, sendo uma das decisões relevantes do empreendedor, tendo cada empresa, de acordo com seu negócio e especificidades, sua demanda potencial. Neste artigo, busca-se dispor de um instrumento para tomada de decisão para locação ótima de empresas com uso da análise multivariada de componentes principais aplicada com método multicritério de otimização. O modelo foi estruturado com a criação de um índice multidimensional, o Índice de Localização Ótima de Empresas - ILOE, através da Análise dos Componentes Principais. A cidade de Fortaleza foi usada para averiguação empírica, representando a complexidade da economia típica dos centros urbanos brasileiros. Deste modo, propõe-se um framework para a decisão do problema de decisão da localização de empresa de varejo, baseado em um conjunto de critérios qualitativos e quantitativos. É um método híbrido de apoio à decisão que combina técnicas do modelo MAUT (Multi-Attribute Utility) com Análise de Componentes Principais, composta de dimensões socioeconômicas presentes no espaço urbano. As evidências empíricas foram verificadas a partir da análise espacial e a congruência da localização de empresas de serviços e comércio atual com o ILOE, num processo de decisão cujas preferências dos decisores foram reveladas.

Palavras-chave: Localização de Empresas. Otimização Multicritério. Análise Multivariada.

Abstract: Among the factors of success of a company, the choice of location is preponderant, being one of the relevant decisions of the entrepreneur, with each company, according to its business and specificities, its potential demand. In this article, we seek to have an instrument for decision-making for optimal location of companies using the multivariate analysis of main components applied with a multicriteria optimization method. The model was structured with the creation of a multidimensional index, the Optimal Company Location Index - ILOE, through the Analysis of the Main Components. The city of Fortaleza was used for empirical investigation, representing the complexity of the economy typical of Brazilian urban centers. In this way, a framework is proposed for the decision of the retail company location decision problem, based on a set of qualitative and quantitative criteria. It is a hybrid decision support method that combines techniques from the MAUT (Multi-Attribute Utility) model with Principal Component Analysis, composed of socioeconomic dimensions present in the urban space. The empirical evidence was verified from the spatial analysis and the congruence of the location of service companies and current trade with ILOE, in a decision process whose preferences of the decision makers were revealed.

Keywords: Business Location. Multicriteria optimization. Multivariate analysis. 


\section{INTRODUÇÃO}

A escolha da localização da empresa é uma das decisões relevantes do empreendedor. Segundo Pereira et al. (2019), a decisão de investimento representa um aspecto típico de estudos locacionais, associado ao caráter de incerteza e inércia que se dá num ambiente de informação imperfeita.

Muitos estudos são direcionados ao apoio à tomada de decisão sobre localização de empresas. Modelos simples de apoio a decisão de localização como o de checklist e modelos estatísticos mais complexos são utilizados a mais de 50 anos (HERNÁNDEZ et al., 1998).

Para Mangini et al. (2017), a localização das empresas é fator de importância na tomada de decisão, de cunho notadamente estratégico, que pode influenciar todo desempenho operacional.

A análise de localização é um processo que envolve o estudo de mercado e o detalhamento de fatores relevantes para o desempenho da organização e, nesse contexto, Almeida (1997) relaciona que fundamentos de geomarketing como técnica para a triangulação de dados, minimização de riscos e incertezas, além de levar em consideração outros fatores de cunho mercadológico, é importante no processo de escolha da localização.

O geomarketing é um ramo da geografia aliada a conceitos de mercado, baseado no processamento de informações geográficas (MELO, 2003). O uso dessas ferramentas torna-se um grande aliado para se conhecer o mercado, seu tamanho e, assim, sua área de influência.

A decisão sobre localização é comumente praticada no corpo gerencial das empresas que, na maioria das vezes, não está preparado para lidar com decisões de localização de instalações, tal a especificidade da questão ou pelos requisitos técnicos necessários para um tratamento mais especializado do problema.

A escolha do local de implantação de uma empresa pode ser feita a partir de várias técnicas e métodos. Dentre as opções, as Análises Multicritérios de Decisão conferem clareza e transparência ao processo de tal maneira que minimiza a incerteza e o risco inerentes à tomada de decisão (MANGINI et al., 2017).

Problemas com múltiplos objetivos e critérios são geralmente conhecidos como Problemas de Otimização Múlti-critérios (Multiple Critéria Optimization) ou de 
Tomada de Decisão Multi-critérios (Multiple Critério Decision Making - MCDM), onde apresentam soluções eficientes que possam espelhar melhor a realidade, enfatizando o estudo dos problemas com múltiplos objetivos (GOMES; GOMES, 2019).

Com modelos multicritérios, pode-se compreender e identificar os critérios fundamentais para selecionar alternativas e podem evitar tomar essas decisões com base apenas na experiência ou feeling individual.

Em estudo baseado na Utilidade Multi-Atributo (MAUT), De La Vega et al. (2017) observaram que essa abordagem possibilita uma análise robusta da decisão mais adequada de acordo com as preferências e aversão ao risco dos tomadores de decisão da empresa, considerando um conjunto de critérios que são avaliados simultaneamente.

A abordagem de Multi-Attribute Utility (MAUT) é desenvolvida para auxiliar na classificação, seleção e/ou comparação de alternativas dentro de um conjunto finito de critérios, de forma que eles se sintam confortáveis com a decisão final (CHEN et al., 2008).

Propõe-se, neste trabalho, um framework para a decisão do problema de decisão da localização de empresa de varejo baseado em um conjunto de critérios qualitativos e quantitativos. É um método híbrido de apoio à tomada de decisão que combina técnicas do modelo MAUT com análise multivariada de componentes principais, composta de dimensões socioeconômicas presentes no espaço urbano.

O objetivo é dispor de um instrumento para tomada de decisão para locação ótima de empresas. O modelo foi estruturado com a criação de um índice multidimensional, o Índice de Localização Ótima de Empresas - ILOE, através da Análise dos Componentes Principais. A cidade de Fortaleza foi usada para averiguação empírica, quinta maior cidade do país com a complexidade da economia típica dos centros urbanos do Brasil.

A opção pelo uso do Multi-Attribute Utility (MAUT) foi para incorporar ao problema múltiplos objetivos e as preferências dos decisores. Ainda, usar um método discreto com número de alternativas discretas na escolha, empregando-se pesos e notas a atributos com uso de uma função matemática. Os atributos referemse às características, tópicos de gestão e localização considerados relevantes pelos decisores, revelando assim, suas preferências. 
Após essa introdução, há o referencial teórico na seção 2. Na seção três a metodologia e, na seção 4, os resultados, incluindo as varáveis qualitativas com o Índice de Localização Ótima-ILOE e o modelo multicritério. Na seção 5 serão analisadas evidências empíricas do modelo com as conclusões na seção 6 .

\section{REFERENCIAL TEÓRICO}

\subsection{Localização da Empresa e Geomarketing}

As diversas Teorias de Localização clássicas são estruturais para o ponto de partida para aplicabilidade no contexto atual dos espaços urbanos, como a teoria do Estado Isolado, Localização Industrial, Localidades Centrais e Lugares Centrais. Elas estão no contexto da geografia econômica, focando na maioria das vezes, na maximização do lucro da empresa (SANTOS, 2004; SILVA, 1987; SILVA, 1976). Essas teorias tomam a indústria como ponto de partida, deixando uma lacuna para definição de localização de empresas de varejo.

Há lugares, espaços, com poder de atração que direcionam as pessoas em direção a eles, podendo ser locais de trabalho, compras ou recreação. O conjunto dessas atividades forma centros que atraem pessoas e podem ser chamados de centros gravitacionais.

Para Brito e Spejorim (2012), o problema da localização depende especificamente da empresa e de suas características. Assim, as empresas, dadas as particularidades, devem verificar as limitações, aptidões das regiões, a identificação das características individuais do local para, baseada nesse conhecimento, definir qual a melhor localização (MATTEI; MATTEI, 2017).

A boa localização da loja também pode ser analisada por: (a) a quantidade de fluxo de tráfego de consumidores relevante, seja tráfego de pedestres ou de veículos; (b) parques de estacionamento; (c) composição da loja; (d) local específico; (e) termos de ocupação; (f) acessibilidade; (g) tempo de viagem; $(\mathrm{h})$ conveniência de localização; (i) outras lojas gratuitas presentes na bacia (GANESHA et al., 2020).

O conhecimento do cenário de um local está relacionado com o geomarketing a partir de informações da interação humana no espaço geográfico. Relaciona-se a 
cartografia, a geografia e o marketing, pois essas disciplinas são fundamentais para o estudo e interações do espaço com as variáveis econômicas e comportamentais humanas (CAVION; PHILIPS, 2006).

Aragão (2004) postula que geomarketing é a nomenclatura empregada no gerenciamento de informações de cunho espacial cujo cerne é o auxílio na tomada de decisão dentro de um cenário mercadológico que permite elencar características de uma região e prover análise do potencial socioeconômico.

Se uma empresa depende do tráfego do cliente (e de fato é), ela precisa realizar uma pesquisa de mercado para restringir a melhor seleção de site com base na pesquisa, nos dados e na análise de alguns determinantes importantes do geomarketing (AKPINAR et al., 2017).

Os GIS-Sistemas de dados georreferenciados, possibilitam a segmentação do mercado por perfil dos consumidores, e também a indicação dos possíveis locais de varejo para atender estes consumidores (BAVIERA-PUIG et al., 2016).

A localização geográfica não deve se apresentar somente como uma localização física no território, mas também algo que apresente uma visão geral da empresa, os seus valores, missão, que seja adaptável às necessidades locais e ao comportamento e demografia do consumidor alvo da região (SAKR; DALLABRIDA, 2017).

De forma empírica ou científica, dois pontos são levados em consideração na tomada de decisão sobre localização: A segmentação e a área de influência.

Conforme Engel et al. (2000), segmentação é o processo de projetar ou caracterizar um produto ou serviço que terá uma atração especial em uma parte do mercado total. Kita et al. (2017) mostram que a identificação e a segmentação de mercado, com base em informações econômico-demográficas, ao considerar um maior número de informações, podem tornar o processo de tomada de decisão da localização do varejo mais racional.

O local cujas características são atrativas de localização e permite mobilidade, atrai um número cada vez maior de empresas e clientes. Torna-se o local aonde um serviço ou produto se torna acessível aos clientes potenciais. A área de influência pode ser conceituada como o poder de atração que passa a ter ou gerar um espaço a partir da instalação de uma empresa, compondo os centros gravitacionais. 
A área de influência representa a extensão máxima que a loja atinge ou, por outro lado, que o consumidor estaria disposto a percorrer para obter um produto ou serviço. Para Sílvia e Parente (2009), o limiar apresenta uma certa variabilidade para os tipos e classes de consumidores, sendo necessário conhecimento e a determinação dos raios de influência de atração por loja e as variáveis que contribuem positivamente e negativamente.

\subsection{Modelo Multicritério de Apoio à Decisão}

Tomar a decisão sobre a localização da empresa requer por parte dos decisores mais do que o simples feeling negocial. Um processo de decisão sobre localização requer um arcabouço robusto de métodos científicos que minimizem os erros e potencializem os acertos da decisão.

Segundo Barros et al. (2017), devido à importância do empreendedorismo, estão sendo usadas técnicas multicritério para avaliar as percepções e tratar dados. Para Briozo e Musetti (2015), a complexidade verificada nas últimas décadas decorrente do aumento do número de informações e da necessidade de utilizá-las no processo decisório fez com que surgissem novos métodos para localização de instalações na tentativa de alcançar maior assertividade nas tomadas de decisão.

Conforme Soares et al. (2020), o Apoio multicritério a Decisão é uma atividade na qual, através de métodos explícitos, mas não necessariamente inteiramente formalizados, ajuda a obter subsídios de respostas às questões colocadas por um decisor no processo de tomada de decisão.

Decisão em grupo e apoio multicritério para tomada de decisão formam duas ferramentas importantes para o processo decisório que envolva dois ou mais decisores. Rhim et al. (2003) investigam como as empresas devem selecionar o número, os locais e a capacidade de suas instalações produtivas.

Como exposto em Jannuzzi et al. (2009), a Análise Multicritério é uma técnica quali-quantitativa, separando as abordagens puramente exploratórias e pouco estruturadas de tomada de decisão, como Brainstorm e Grupos de Discussão, e os modelos quantitativos rigidamente estruturados da Pesquisa Operacional.

Técnicas e modelos mais sofisticados foram construídos, tais como, paretoótimo, desenvolvido para tratar dos problemas multiobjectivos. A "solução eficiente" 
de Pareto é aquela que pode ser obtida de forma que a alternativa escolhida atinja um valor amplo em todos os critérios e não possua um decréscimo simultâneo (um valor dominado por outra alternativa) em nível inaceitável em qualquer dos demais critérios que estão sendo utilizados no processo de avaliação das alternativas (GOMES; GOMES, 2019).

Muitas vezes, no processo de montagem do problema, observam-se múltiplos objetivos, como buscar uma localização como muito fluxo de pessoas e com mobilidade urbana. Ao mesmo tempo, esses objetivos podem ser conflitantes, exemplo: locais com muito fluxo de pessoas geralmente tem pouca mobilidade.

O problema de maximizar ou minimizar múltiplos objetivos pode ser expresso com um vetor de variáveis de decisão cujos elementos representam as funções objetivos. Os problemas com múltiplos objetivos possuem um conjunto de solucões ótimas que estão na fronteira Pareto-ótima, mas decidir qual melhor solução passa pelo processo multicritério que visa escolher, entre as várias alternativas eficientes, aquela que for mais satisfatória.

Chacón-García (2017) mostram que a combinação de métodos de análise espacial e métodos de tomada de decisão multicritério, ao ordenar as alternativas (locais) conforme critérios de mercado, podem reduzir riscos de localização.

No MAUT (Multiple Attribute Utility Theory), a função objetivo é a representação matemática do critério de eficiência adotado no problema de otimização. É influenciada pelas variáveis de projeto, conhecidas como variáveis de decisão do problema. Esse critério pode ser representado por uma única função objetivo, no caso de problemas mono-objetivos, ou por várias funções objetivo, no caso de problemas multiobjectivos (GOMES; GOMES, 2019).

Modelos multi-objetivos fornecem flexibilidade no processo de otimização que não pode ser alcançada por modelos mono-objetivos. O grau de liberdade leva a um conjunto de soluções que são chamadas de soluções de Pareto (AMIN; BAKI, 2017).

Para Ehrlich (1996), o MAUT parte da construção de utilidades individuais para cada critério e, dentro deste, quantifícar e ordenar as preferências através da agregação dos diversos critérios em uma única função de utilidade que comporta a importância de cada critério, além das substituições (tradeoffs) e dos seus relacionamentos. 
Conforme Nunes e Silva (2019), o MAUT surgiu como derivação da teoria da utilidade esperada e incorporou a questão do tratamento de múltiplos objetivos (denominados nesta teoria de atributos).

Um problema de decisão multicritério consiste numa situação em que há pelo menos duas alternativas de ação para se escolher, e essa escolha é conduzida pelo desejo de se atender a múltiplos objetivos, muitas vezes conflitantes entre si (ALMEIDA, 2013). Para Ester et al. (2018), esse modelo consiste na determinação de uma relação de fatores relevantes para a decisão de localização, com a atribuição de notas por meio de uma escala arbitrária.

\section{METODOLOGIA E MÉTODOS}

\subsection{Dimensões que definem as Áreas Potenciais de Negócios}

A metodologia usada é composta pela definição de um índice que define espacialmente a localização potencial de negócio e delimita o espaço para que as preferências dos decisores sejam utilizadas num método multicritério de otimização da decisão. Os fatores segmentação e área de influência são relevantes na decisão da localização, assim, elencam-se três dimensões que devem ser visitadas para a definição da localização: Dimensão Mercado Potencial, Dimensão Espacial e Dimensão Econômica.

Neste artigo o foco serão as classes C e D, definidas, por suposição, como os clientes potenciais de determinadas médias e pequenas empresas de varejo. Assim, um dos objetivos é definir um índice para mostrar os espaços geográficos urbanos onde se concentram esse grupo populacional de clientes potenciais.

Para uma empresa nova abrir ou expandir seus negócios se faz necessário conhecer o tamanho de seu mercado, dos clientes em potencial. Isso se faz pelo estudo de mercado, assim, onde deverá ser a localização do projeto envolve, dentre outros fatores, a demanda potencial para determinados negócios inerentes ao setor de serviços e comércio. Um ponto importante para a localização da área de serviços e comércio é a proximidade ao mercado consumidor.

O ramo da geografia aliada a conceitos de mercado, baseado no processamento de informações geográficas, é o geomarketing. A Dimensão Espacial 
refere-se ao local geográfico, na percepção espaço-temporal dos clientes em potencial. Dado que são as classes "C" e "D" as mais compatíveis com os consumidores potenciais por suposição para esse trabalho, são esses espaços onde essas classes residem e onde se locomovem que devem ser definidos como áreas prioritárias. Busca-se inserir o critério de onde está o cliente potencial, cujas áreas deverão ter as seguintes características:

- Lugar de domicílio das pessoas dessas classes sociais;

- Que sejam densas em população, mas não tanto que se caracterize como favelas (pois os clientes focados não estão concentrados nelas);

- Espaços equidistantes, próximos de centros comerciais dos bairros e das residências em uma área não superior a $1 \mathrm{~km}^{2}$ (aqui entra a suposição que $1 \mathrm{~km}^{2}$ é a área de influência);

- Local de cluster comerciais/serviços, que são os centros comerciais e serviços.

Outro fator importante do espaço é seu polígono de atuação e de atração, já exposto como área de influência. Parte-se da suposição que a área de atuação e atração deverá ter em média de $1 \mathrm{Km}^{2}$. Essa suposição vem da visualização empírica da convergência entre a população focal e a concentração de edificações comerciais numa área (buffer) de aproximadamente $1 \mathrm{~km}^{2}$. As pessoas dessa classe social estão comprando cada vez mais próximo de suas residências, sinalizando que elas estão buscando mais conforto, mobilidade, segurança e confiança.

Com relação ainda há área de influência, a partir de uma amostra de 5 lojas de um mesmo grupo, Parente e Kato (2001) verificaram que $60 \%$ dos clientes da loja 1 ficam dentro do raio de $1 \mathrm{~km}$. Esse valor (60\% a $70 \%$ ) coincide com o que tem sido utilizado para determinar a área de influência primária. A sugestão deste estudo é operacionalizar o conceito da área de influência como dentro do raio que contém $60 \%$ dos clientes. Aqui os autores usam o raio de $1 \mathrm{~km}$ que dá aproximadamente 3 $\mathrm{Km}^{2}$.

Classe social geralmente é o elemento essencial para a busca de clientes. Para definir o produto, qualidade, dentre outros itens, deve-se antes ter a resposta para: qual meu cliente? A classe social e a definição das pessoas que nela estão é um bom caminho para o critério da busca. 
A visão governamental das classes sociais, utilizada pelo IBGE no censo populacional a cada dez anos, é baseada no número de salários mínimos. Mais simples, divide em apenas cinco faixas de renda ou classes sociais, conforme o Quadro 1.

Quadro 1 - Classificação das Cl
Sociais - IBGE
\begin{tabular}{|l|l|}
\hline CLASSE & SM \\
\hline A & $\begin{array}{l}\text { Acima de } \\
\text { SM }\end{array}$ \\
\hline B & 10 a 20 SM \\
\hline C & 4 a 10 SM \\
\hline D & 2 a 4 SM \\
\hline E & até 2 SM \\
\hline
\end{tabular}

Fonte: IBGE

Procura-se criar um índice que capture a classe social não apenas pela renda, mas também pelo consumo e pela área geográfica estabelecida como domicílio. São as classes C e D que serão utilizadas neste trabalho, ou seja, no intervalo de 2 a 10 salários mínimos.

\subsection{Descrição dos Dados e do Espaço}

Para compor as variáveis utilizadas nas dimensões mercado, econômica e espacial, buscou-se no censo demográfico de 2010 as variáveis proxy (relacionadas com as variáveis não observadas) que possam capturar o perfil das dimensões relacionadas, principalmente com as classes sociais " $C$ " e "D". Esta pesquisa de utilizou da base digital do mapa de setores censitários do ano de 2010, que dividiu a cidade de Fortaleza em 3.043 setores censitários. Os dados relativos ao censo foram obtidos por meio de download do sítio oficial do IBGE (http://www.ibge.gov.br).

As variáveis escolhidas do censo referem-se a proxy de comportamento de consumo e renda das classes sociais. Renda per capta é importante para verificar a distribuição da renda dada a população existente em cada área (setor censitário). Restringiu-se aos valores de renda entre 3 a 10 salários mínimos, tanto no quantitativo de pessoas com essa renda como na per capta, além do total de 
rendimentos. Outra variável com poder de explicação é a quantidade de banheiros exclusivos por domicílios, indicando o poder aquisitivo entre as classes social pela escolha do nível da residência. Foram selecionadas, para a construção do índice, as variáveis do Quadro 2.

Quadro 2 - Descrição das Variáveis Usadas no Modelo

\begin{tabular}{|l|l|}
\hline \multicolumn{1}{|c|}{ Variável } & \multicolumn{1}{|c|}{ Descrição } \\
\hline Ren_Percap & $\begin{array}{l}\text { Média do rendimento nominal mensal per capita nos domicílios } \\
\text { permanentes }\end{array}$ \\
\hline Pes $>10 ~ 3-5-s m$ & $\begin{array}{l}\text { Pessoas de }>=10 \text { anos de idade com rendimentos nominal mensal de } \\
3-5 \mathrm{SM}\end{array}$ \\
\hline Pes $>10$ 5-10-sm & $\begin{array}{l}\text { Pessoas de }>=10 \text { anos de idade com rendimentos nominal mensal de } \\
5-10 \mathrm{SM}\end{array}$ \\
\hline tt_rend 3-5-sm & $\begin{array}{l}\text { Total de rendimentos nominal mensal das pessoas de 10 anos ou mais } \\
\text { de idade com rendimentos nominal de 3 a 5 SM }\end{array}$ \\
\hline tt_rend 5-10-sm & $\begin{array}{l}\text { Total de rendimentos nominal mensal das pessoas de 10 anos ou mais } \\
\text { de idade com rendimentos nominal de 5 a 10 SM }\end{array}$ \\
\hline 2wc & $\begin{array}{l}\text { Domicílios permanentes com 2 banheiros de uso exclusivo dos } \\
\text { moradores }\end{array}$ \\
\hline 3 wc & $\begin{array}{l}\text { Domicílios permanentes com => 3 banheiros de uso exclusivo dos } \\
\text { moradores }\end{array}$ \\
\hline Rend-perc- 3-5 sm & $\begin{array}{l}\text { Domicílios particulares rendimento nominal domiciliar per capita de 3 a } \\
5 \text { SM }\end{array}$ \\
\hline $\begin{array}{l}\text { Rend-perc-5-10 } \\
\text { sm }\end{array}$ & Domicílios com rendimento nominal domiciliar per capita de 5 a 10 SM \\
\hline
\end{tabular}

Fonte: Elaboração do autor

\subsection{Análise Multivariada de Componentes Principais}

Para construção do índice de localização ótima, utilizou-se a análise fatorial na busca de fatores dos componentes principais que explicassem e representassem a variabilidade comum das variáveis elencadas no Quadro 2 para o Município de Fortaleza.

A análise fatorial tenta capturar o que se convencionou chamar de variáveis latentes. Estas são o elo comum entre variáveis que comungam algumas características, de algum tipo de relacionamento que provoca variações comuns entre elas. Para Corrar et al. (2014), a análise fatorial é uma técnica estatística que busca, através da avaliação de um conjunto de variáveis, a identificação de dimensões de variabilidade comuns existentes em um conjunto de fenômenos.

Para verificar se as variáveis são compatíveis em relação a variabilidades comuns para se fazer a Análise de Componentes Principais da Análise Fatorial, o 
Teste de KMO e Bartlett são usados. Com uso do software estatístico Statistical Package for the Social Sciences (SPSS) - IBM, foram feitos os testes e análise fatorial.

A ideia é criar o índice com todos os fatores relevantes, ponderando-os usando como pesos os respectivos autovalores. Após a estimação das cargas fatoriais e dos escores fatoriais e utilizando-se da Equação 1, será criado a média ponderada para cada setor agregado, chamada de Índice Bruto.

$$
I B=\frac{\sum_{i=1}\left(w_{i} F_{i}\right)}{\sum_{i=1} w_{i}}
$$

Sendo:

IB = Índice Bruto

$\mathrm{W}_{\mathrm{i}}=$ proporção da variância explicada por cada fator

$\mathrm{Fi}_{\mathrm{i}}=$ escores fatoriais

Após a construção do índice, o mesmo é padronizado utilizando-se da equação abaixo, provocando média zero e desvio padrão igual a um. O objetivo é transformar o índice para ser autoexplicativo e ordinal do tipo quanto maior, melhor a localidade(espaço) para a empresa. $O$ índice estará no intervalo entre 0 e 1. Quanto mais próximo de 1 mais próximo dos extremos e quanto mais próximo de zero, mas próximo do extremo menor.

$$
\text { Îndice }=\frac{X-I B_{\operatorname{Min}}}{I B_{\operatorname{Max}}-I B_{\operatorname{Min}}}
$$

Sendo:

X: o valor do índice bruto do setor agregado - IB;

IBmin: o valor do setor agregado de menor índice bruto e;

IBmax: o valor do setor agregado de maior índice bruto 


\section{RESULTADOS}

\section{1 Índice de Localização Ótima de Empresas - ILOE - Construção e Representação Espacial}

As nove (9) variáveis utilizadas na construção do índice foram condensadas em 2 fatores, onde cada fator engloba as variáveis que apresentam um padrão parecido. No método de componentes principais, a medida para mostrar o quanto os fatores explicam a variância total dos dados é o KMO que, nesse conjunto de dados se mostrou satisfatório com valor de 0,877 .

\begin{tabular}{lll} 
Tabela 1 - Teste de KMO e Bartlett & & \\
\hline Medida Kaiser-Meyer-Olkin de adequação de amostragem. &, 877 \\
Teste de esfericidade de Bartlett & Aprox. Qui-quadrado & 70240,255 \\
& gl & 36 \\
& Sig. & 0,000
\end{tabular}

Fonte: Resultado da Análise de Componentes Principais - SPSS

Após o processo fatorial, observa-se pela tabela de variância explicada total na Tabela 2 que dois fatores(componentes) relevantes foram encontrados, Fator $1 \mathrm{e}$ Fator 2, ambos juntos com o poder de explicar a variabilidade das variáveis em $91,5 \%$.

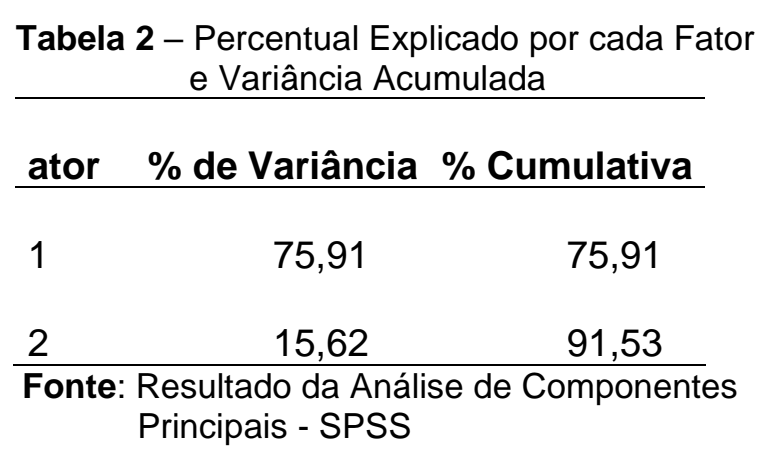


A Tabela 3 mostra as variáveis que foram inseridas em cada fator. Juntadas pela análise fatorial de componentes principais conforme o grau de correlação entre elas.

Tabela 3 - Cargas Fatoriais*

\begin{tabular}{|c|c|c|}
\hline Variável & F1 & F2 \\
\hline rend_percap & 0,892 & 0,049 \\
\hline pes $>10 \_$de 3_5_sm & 0,570 & 0,780 \\
\hline pes $>10 \_$de 5_10_sm & 0,903 & 0,403 \\
\hline tt_rend_3_5_sm & 0,583 & 0,773 \\
\hline tt_rend_5_10_sm & 0,917 & 0,366 \\
\hline $2 w c$ & $-0,071$ & 0,915 \\
\hline 3 wc & 0,933 & 0,175 \\
\hline renda_perc_de 3_5 sm & 0,819 & 0,515 \\
\hline renda_perc_de 5_10 sm & 0,959 & 0,131 \\
\hline \multicolumn{3}{|c|}{ Matriz de componente rotativa (Rotação convergida em 3 iterações) } \\
\hline \multicolumn{3}{|l|}{ Método de Extração: Análise de Componente Principal. } \\
\hline Método de Rotação: Varimax com Normalização de Kaiser & & \\
\hline
\end{tabular}

Para a construção do Índice de Localização Ótima-ILOE, em virtude da importância dos dois fatores extraídos, os mesmos foram ponderados usando como pesos a variância contida nos respectivos autovalores da Tabela 2 e com uso da equação 1. Além disso, o mesmo foi padronizado utilizando-se da equação 2, o que transformou o índice no intervalor de 0 a 1 . Quanto mais próximo de 1 mais próximo dos extremos da classe social "C" e quanto mais próximo de zero, mas próximo do extremo menor da classe social "D" ou "E".

Quanto mais próxima de 1, melhor a condição econômico-social daquela área geográfica, utilizados aqui os setores censitários. Para Fortaleza, cujos dados do IBGE foram utilizados para essa capital, o IBGE utilizou 3.020 setores censitários. Para cada setor censitário foi definido um ILOE. Para melhor compreensão dos espaços, dividiu-se em quartil iguais todos os setores, assim, os setores de cores verde nas Figuras 1 e 2 de Fortaleza demonstram a localização das classes "C" e "D", em cores vermelha e laranja da Figura 1 estão as classes menos favorecidas. 


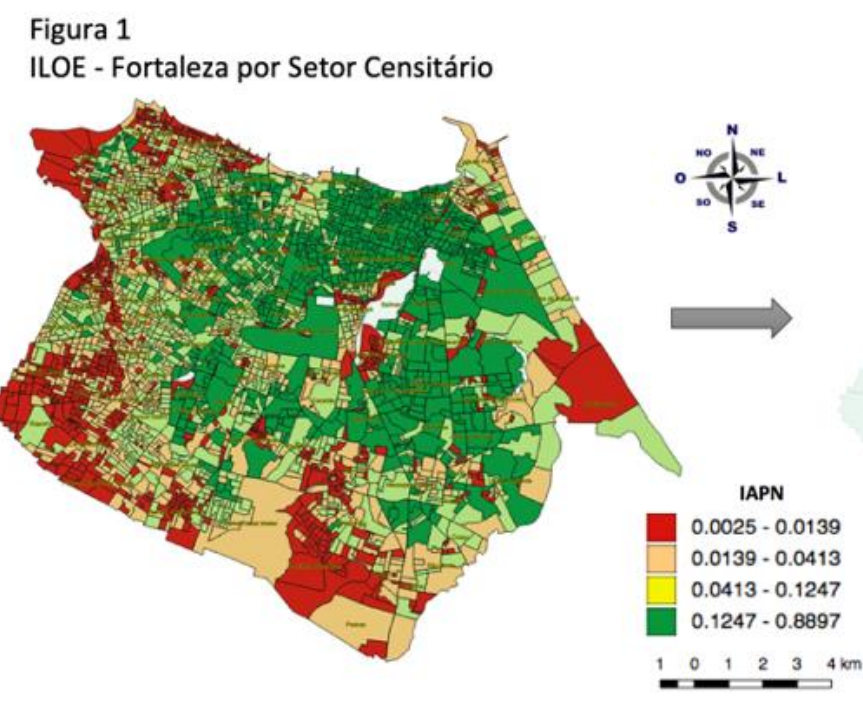

Fonte: Elaboração do Autor.

Na Figura 1 toda Fortaleza está mapeada com a distinção das cores de acordo com o ILOE, onde o verde com melhor área potencial e as áreas vermelhas sem potencial mercadológico para o foco desse trabalho. Na Figura 2, ressalta-se só os setores censitário relacionados com as melhores condições socioeconômicas das classes "C" e "D". Dos 3.020 setores censitários, ficaram os 756 setores em verde na Figura 2.

Para mais uma triagem, utiliza-se novamente o quartil estatístico para os 756 setores (em verde na Figura 2), dividindo-os novamente em quatro quartis onde 0 quartil de melhor ILOE são os de cor verde conforme Figura 4. Na Figura 3 encontram-se todas as 756 áreas pela classificação potencial das classes "C" e "D". Para classificar mais os espaços nas áreas de maiores potenciais de negócio, separam-se os 189 setores censitário em cor verde conforme Figura 4 com alto potencial de clientes. A soma dessas áreas potenciais em verde equivale a $31,3 \mathrm{Km}^{2}$ representando apenas $9,8 \%$ do território de Fortaleza. 


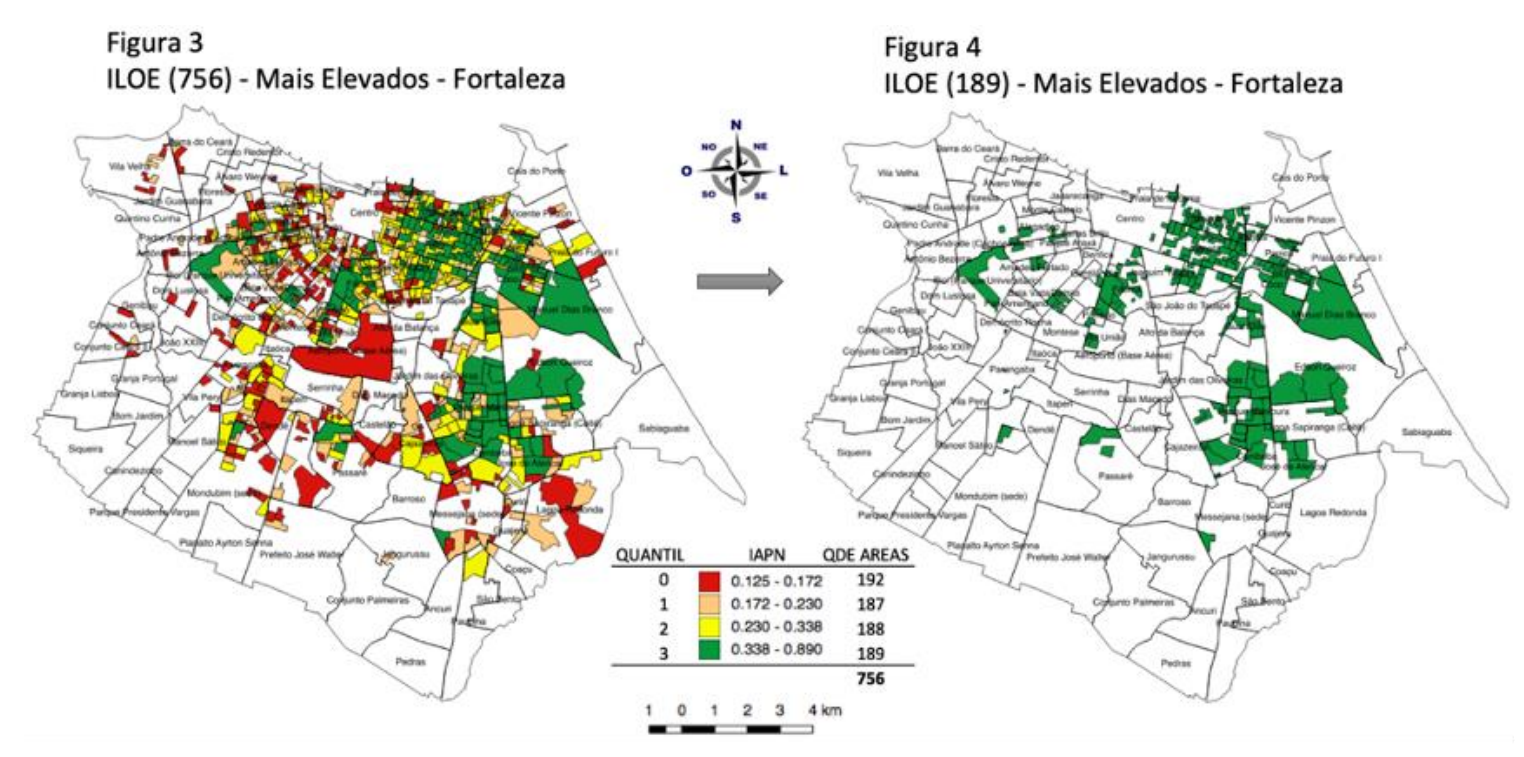

Fonte: Elaboração do Autor.

Salienta-se que as classes sociais "C" e "D" encontram-se dispersas em toda Fortaleza, mas com maior concentração nas 756 áreas conforme Figura 3. Para classificação de área prioritárias, foram utilizadas as 189 áreas em cor verde conforme Figura 4. O objetivo é responder à pergunta: Quais áreas principais devem priorizar? As 189 áreas de maior potencial conforme Figura 4. A partir de agora, essas 189 áreas serão chamadas de ILOE (189).

A partir do Índice de Localização Ótima de Empresas-ILOE, chega-se as áreas geográficas em torno $0,4 \mathrm{Km}^{2}$ por setor, em que as áreas em verde têm o ILOE mais alto, logo, de maior potencial de negócio.

\subsection{Modelo Multicritério x Índice de Localização Ótima - ILOE}

A tomada de decisão multicritério está firmemente baseada no conceito de otimalidade onde múltiplos critérios caracterizam a noção de "melhor". Aspectos relacionados à qualidade devem ser observados concomitantemente as análises de engenharia econômica para localização, como aspectos relacionados à satisfação do cliente, comodidade, mobilidade, segurança, dentre outros fatores. Critérios para as variáveis qualitativas inerentes à localização são incorporados.

As alternativas são confrontadas e avaliadas pelas variáveis de decisão que, mapeadas pelos atributos, representam as preferências dos decisores por critérios relacionados aos atributos. O problema então é definir, conforme o fluxo da 
construção do modelo da Figura 5: i) os objetivos; ii) as variáveis de decisão; iii) os atributos de preferência dos decisores, e iv) Critérios para os atributos.

Figura 5 - Fluxo da Construção do Modelo Híbrido: MAUT mais ILOE

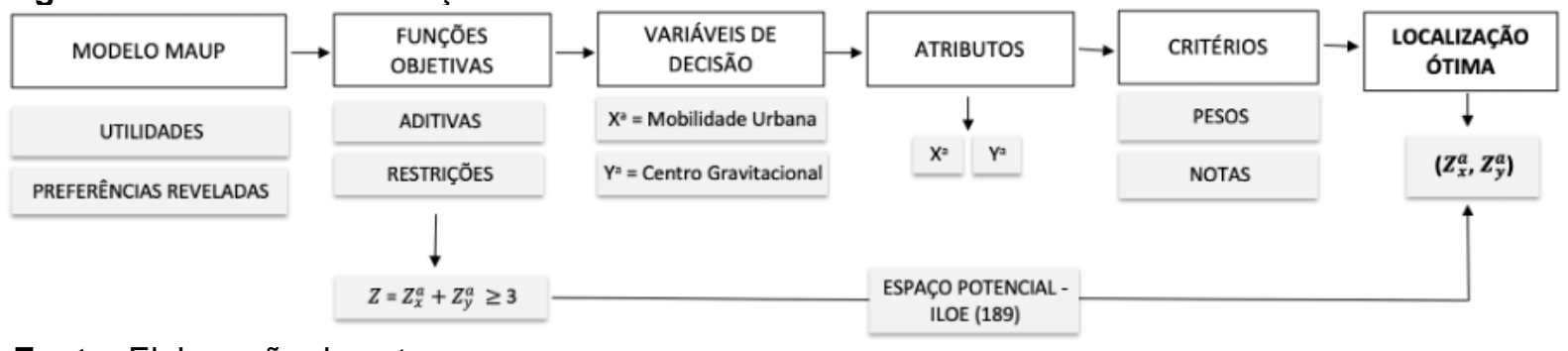

Fonte: Elaboração do autor.

Uma vez definidos os espaços urbanos potenciais cuja localização foi delimitada pelo ILOE, incorpora-se a essas localizações, em torno de 0,4 km²(área média dos setores censitários), aspectos qualitativos de localização através de um modelo de decisão multicritério. Conforme as preferências do decisor relacionados à localização ideal, pondera-se atributos e critérios. O Índice de Localização ÓtimaILOE define um espaço, setor censitário, de potencial de negócio, mas não define a localização exata, em qual rua deve ser colocada empresa. Deste modo, a partir do setor censitário definido pelo ILOE, os decisores, a partir de critérios definidos a priori, definem atributos e pesos que irão classificar por rua naquele setor para se obter a escolha. As ruas são as alternativas a serem classificadas e objeto de decisão.

Um problema de decisão multicritério envolve os seguintes elementos: Conjunto de alternativas, que são opções para possíveis soluções; conjunto de atributos, assim, as alternativas podem ser comparadas entre si e, o conjunto de critérios, servindo para representar cada ponto de vista no qual as comparações são realizadas.

Com uso da Teoria da Utilidade Multicritério (Multiple Attribute Utility Theory MAUT) na construção de soluções ótimas, definem-se indicadores de localização potencial. Cada indicador é um atributo, estacionamento no local ou vizinhança, rua com boa estrutura física, fluxo intenso de pessoas, dentre outros fatores relevantes para uma boa localização empresarial. Então pode-se definir como variáveis de decisão, aspectos de localização relevantes para o decisor tais quais: 


$$
\begin{aligned}
& X^{\mathrm{a}}=\text { Mobilidade Urbano } \\
& Y^{\mathrm{a}}=\text { Centro Gravitacional }
\end{aligned}
$$

Cada função objetivo com suas respectivas variáveis de decisão têm atributos (Quadro 1) que pertencem ao vetor de decisão. As preferências subjetivas dos decisores entre as alternativas são mensuradas ou reveladas pela ponderação de vários critérios. Cada rua dentro do espaço de busca, delimitado pelo ILOE, que disputa a preferência do decisor, terá seu $X^{\mathrm{a}}$ e $Y^{\mathrm{a}}$ mensurados. As funções objetivas representam as preferências relativas do decisor entre os atributos de um conjunto.

As variáveis de decisão referem-se às decisões a serem tomadas, visando encontrar a solução do problema. Para parametrizar estas variáveis serão definidos critérios conforme as preferências do decisor, este parametriza as variáveis de decisão com os atributos constantes no Quadro 3 e critérios de pesos conforme Quadro 4. Trata-se de modelo de decisão a priori, o gestor é consultado uma única vez, antes do início do processo de otimização e a informação obtida quanto aos seus interesses é usada para guiar a busca pela solução favorita pertencente à fronteira Pareto.

Quadro 3 - Atributos das variáveis de Decisão
\begin{tabular}{|l|l|}
\hline Atribuição dos pesos aos critérios dos atributos \\
\hline i & Mobilidade Urbana \\
\hline 1 & Estacionamento no local ou vizinhança \\
\hline 2 & Rua com boa estrutura física \\
\hline 3 & Tráfico de carros intenso mais fluente \\
\hline 4 & Proximidade de metrô e/ou terminal de ônibus \\
\hline i & Centro Gravitacional \\
\hline 1 & Fluxo intenso de pessoas \\
\hline 2 & Proximidade de polo de comércio e/ou serviço. \\
\hline 3 & Segurança da área e entorno \\
\hline
\end{tabular}

Fonte: Elaboração do autor 
Os decisores definiram os atributos no Quadro 3 com os pesos para cada critério conforme Quadro 4, criando, a partir da soma dos atributos para cada variável de decisão, $X^{a}$ e $Y^{a}$.

Uma vez definido cada atributo das variáveis de decisão, o processo agora é definir a pontuação das alternativas, as ruas(alternativas) que estão concorrendo serão avaliadas e pontuadas em cada atributo, utilizando os pesos do Quadro 4. A pontuação final da rua é dada pela soma final dos atributos de cada variável de decisão, variando de 0 a 10 a respectiva pontuação em $X^{a}$ e $Y^{a}$.

Quadro 4 - Critérios para Atributos (Pesos) das variáveis de Decisão
\begin{tabular}{|l|c|}
\hline Critérios de Pesos para os Atributos & Peso \\
\hline Não Significante & 0 \\
\hline Significante & 1 \\
\hline Muito Significante & 2 \\
\hline
\end{tabular}

Fonte: Elaboração do autor

Dadas as definições dos pesos (Quadro 4) nos atributos do Quadro 3, utilizase o Quadro 5 para reclassificar os pesos em intervalos de escalonamento da soma dos atributos por função objetiva, $f\left(X^{a}\right)$ e $f\left(Y^{a}\right)$. Exemplo: Supondo que a soma dos pesos dos atributos de $X$ seja igual a 8 , então o mesmo será reclassificado pela Tabela 8 para peso 2, pois está no intervalo de 8 a 10. Caso a soma dos atributos de Y seja 6, então será reclassificado para 1, o que levará ao ponto $\left(X^{a}, Y^{a}\right)=(2,1)$ no espaço de decisão.

Quadro 5 - Critérios Intervalar de Escalonamento para Atributos (Pesos) das Funções Objetivas $\mathrm{X}$ e $\mathrm{Y}$

\begin{tabular}{|l|c|}
\hline Critérios & Intervalos da Soma X e Y \\
\hline Localização & De 0 a 4 \\
\hline Boa & De 5 a 7 \\
\hline Excelente & De 8 a 10 \\
\hline
\end{tabular}

Fonte: Elaboração do autor

São duas varáveis de decisão, $X^{a}$ e $Y^{a}$, com três notas $(0,1$ e 2) atribuídos pela Tabela 3, a partir dos pesos sobre os atributos da Tabela 1. A ordem ( $X^{a}$, $\left.Y^{a}\right)$ é relevante. Sendo $X^{a}=2$ e $Y^{a}=1$, então há o ponto $(2,1)$, que é diferente do ponto $(1,2)$. Sendo os atributos independentes que respaldam as duas variáveis de 
decisão $X^{a}$ e $Y^{a}$ com diferença na ordem e que podem ser repetidos $(1,1)$, o problema terá nove alternativas, dado o arranjo com repetição $A(n, p)=n^{p}$.

O modelo é apresentado nas equações (3.1) a (14). $Z$ representa a função utilidade revelando as preferências dos decisores através dos parâmetros de localização ótima, usada para decidir qual rua será escolhida dentro da delimitação imposta pelo ILOE. (3.1) e (3.2) são as funções objetivas, funções constantes conforme intervalos para as variáveis de decisão $X^{a}$ e $Y^{a}$ da alternativa $A$. Então o problema de otimização configura-se:

Maximizar:

$Z_{x}^{a}=f\left(X^{a}\right)=\left\{\begin{array}{l}0 \text { se } 0<X^{a} \leq 4 \\ 1 \text { se } 4<X^{a} \leq 7 \\ 2 \text { se } 7<X^{a} \leq 10\end{array} \quad Z_{y}^{a}=f\left(Y^{a}\right)=\left\{\begin{array}{l}0 \text { se } 0<Y^{a} \leq 4 \\ 1 \text { se } 4<Y^{a} \leq 7 \\ 2 \text { se } 7<Y^{a} \leq 10\end{array}\right.\right.$

$Z^{a}=\left(Z_{x}^{a}, Z_{y}^{a}\right)$

$Z^{a}=\left(f\left(X^{a}\right), f\left(Y^{a}\right)\right)$

$Z^{a}=f\left(X^{a}\right)+f\left(Y^{a}\right)=Z_{x}^{a}+Z_{y}^{a}$

Sujeito às Restrições:

$0<X^{a}, Y^{a} \leq 10$

$X^{a}+Y^{a} \geq 13 \quad \forall X^{a}, Y^{a} \geq 5$

$Z^{a}=f\left(X^{a}\right)+f\left(Y^{a}\right)=Z_{x}^{a}+Z_{y}^{a} \geq 3 \forall Z_{x}^{a}, Z_{y}^{a}>0$

$d_{i}=\left(X_{x}^{a}, Y_{y}^{a}\right) \in D=$ Espaço de Decisão

$Z^{a}=\left(Z_{x}^{a}, Z_{y}^{a}\right) \in Z=$ Espaço de Objetivos

$Z=Z_{x}^{a}+Z_{y}^{a} \geq 3$

$Z_{x}^{a}, Z_{y}^{a} \in \mathrm{N} / 0 \leq Z_{x}^{a}, Z_{y}^{a} \leq 2$

$D=$ Espaço de Decisão $\subset$ ILOE (189)

Para cada solução $d_{i}=\left(X_{x}^{a}, Y_{y}^{a}\right)$ em $\mathrm{D}$, existe um ponto $Z^{a}=\left(Z_{x}^{a}, Z_{y}^{a}\right)$ no espaço objetivo $Z$ conforme figura 6. D está contido no ILOE conforme restrição (14) significando que o mapeamento das ruas no espaço de decisão deverá fazer parte do espaço de localização ótima delimitado pelo ILOE (189) - 189 áreas de maior 
potencial. Em otimização multiobjetivo, o conceito de otimalidade baseia-se no conceito de dominância de pareto. Neste problema específico, as duas funções objetivos especificam critérios de localização ótima em que o aumento da mobilidade urbana num certo nível é inversamente proporcional ao nível do centro gravitacional, muitas pessoas circulando cria problemas de mobilidade urbana, logo, para aumentar $\mathrm{X}$ deverá sacrificar $\mathrm{Y}$ e vice-versa.

A restrição (8) no espaço de decisão equivale a restrição (12) no espaço dos objetivos, significando que o empresário-decisor definiu que as possibilidades de aceitação para localização de uma determinada rua ocorrerá se as variáveis dos atributos, sintetizados pelo Quadro 3, tenham a soma de seus pesos dos critérios no mínimo três(3), o que significa limitar a aceitação a um grau mínimo de mobilidade e nível de centro gravitacional aceitáveis.

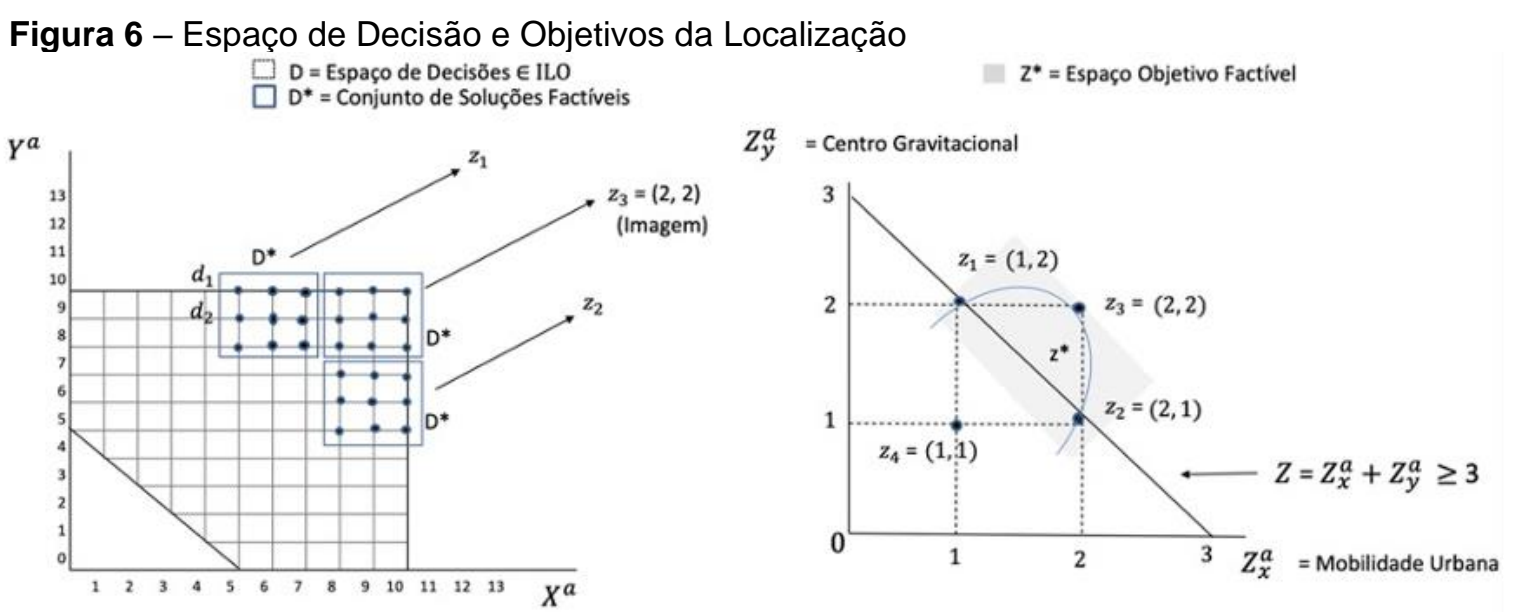

Fonte: Elaboração do autor

O problema aqui exposto tem múltiplos objetivos, traduzidos no vetor $\vec{Z}^{a}$ com indicadores e seus graus de localização aceitáveis e que estão no conjunto de soluções ótimas, denominado fronteira Pareto-ótima ou não-dominada. As funções objetivas equivalem a querer concomitantemente uma localização ótima com $Z_{x}^{a}=$ Centro gravitacional e $Z_{y}^{a}=$ Mobilidade Urbana. Nesse contexto, $Z^{a}=\left(Z_{x}^{a}, Z_{y}^{a}\right)$ é a função de maximização da função dados os critérios traduzidos nos indicadores de localização.

Na figura 6, o Espaço de Objetivos é plotado com as funções que objetivam maximizar o modelo de localização, dada a possibilidade da decisão sobre aspectos 
relevantes de localização definidos a priori. A função objetiva (3) mapeia os pontos exequíveis no espaço de decisão. A restrição (8), como já citado, traduz a restrição para a escolha da localização no nível mínimo de aceitação dos atributos.

Dada a restrição (12), os dois vetores $\overrightarrow{z_{1}}$ e $\overrightarrow{z_{2}}$ plotados na figura 6 , têm por parte do decisor indiferença na preferência. No ponto $(2,2)$ há uma cesta com atributos do grau de localização melhor pois está acima da restrição. Já no ponto (1, 1), há uma cesta com atributos do grau menor, sendo inferior.

Para um vetor $\overrightarrow{z_{k}}$ com soma nos atributos acima de três, $(2,2)>(1,2)$, este será preferível a um com soma $\vec{z}_{i}=3$. Já vetores com somas iguais, como $(2,1) \sim$ (1, 2), $Z_{1} \sim Z_{2}$, significa que o decisor será indiferente na preferência. O ponto(vetor) ótimo $\overrightarrow{z_{3}}=(2,2)$ deverá ser escolhido, significando que a rua(alternativa) com esses atributos é a melhor localização dentro do espaço delimitado pelo ILOE e dadas as preferências do empresário(decisor).

\section{EVIDÊNCIAS EMPÍRICAS: CONVERGÊNCIA ESPACIAL COM OS SETORES DA ECONOMIA}

O objetivo dessa seção é verificar se existe convergência entre as áreas escolhidas através do ILOE e os setores comercial e de serviços. Usa-se esses espaços de densidade dos setores de serviços e comercial como áreas de controle, para fazer um batimento da precisão do ILOE na sinalização das áreas potenciais. A fonte dos dados foi do observatório da Prefeitura de Fortaleza para o ano de 2015 (https://observatoriodefortaleza.fortaleza.ce.gov.br.).

\section{1 Índice de localização ótima de Empresas versus Edificações Comerciais}

As áreas (189) escolhidas através do ILOE estão nos bairros onde há maiores metragens de edificações comerciais de Fortaleza, o que ratifica a veracidade deste índice para sinalizar áreas potenciais de negócio, conforme Figura 7. 
Figura 7 - 189 ILOEs Altos X Áreas Comerciais por Bairro de Fortaleza

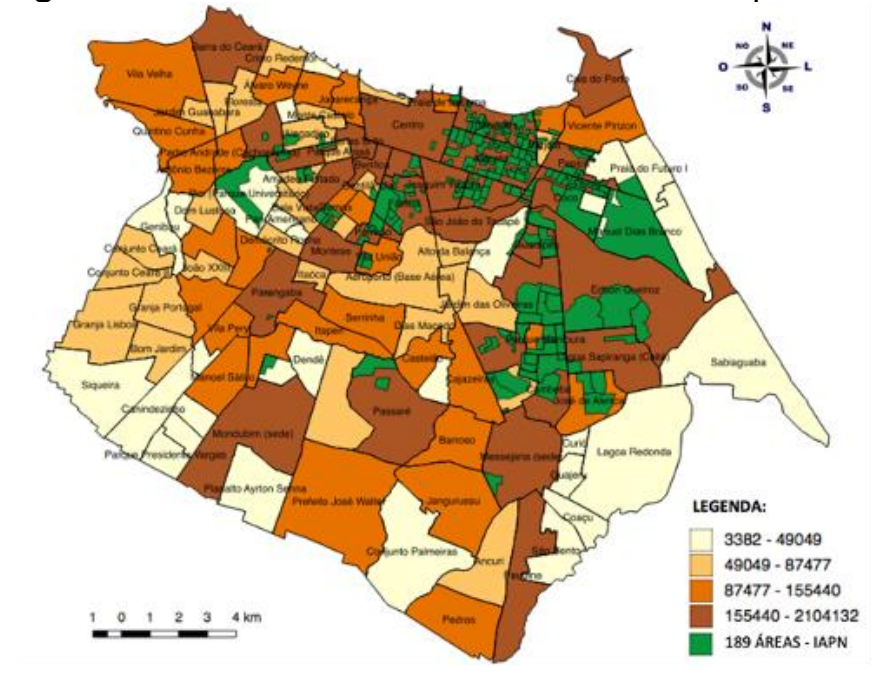

Fonte: Elaboração do autor

As áreas em marrom são os bairros de Fortaleza com maiores metragens de edificações comerciais. Nesses bairros mais comerciais, é observado que as áreas potenciais de negócio espelhados pelo ILOE, principalmente para as áreas mais bem classificadas, representadas pela cor marrom escuro, estão instaladas espacialmente exatamente nesses bairros.

\section{2 Índice de Localização Ótima de Empresas Versus Edificações de Serviços}

Tal qual aos bairros comerciais, a classificação das áreas por serviços, podem ser observadas na Figura 8, que as áreas escolhidas através do ILOE estão exatamente nos bairros onde temos maiores metragens de edificações de serviços em Fortaleza, o que ratifica a veracidade deste índice para sinalizar áreas potenciais de negócio. 
Figura 8 - 189 ILOEs Altos X Áreas Serviços por Bairro de Fortaleza

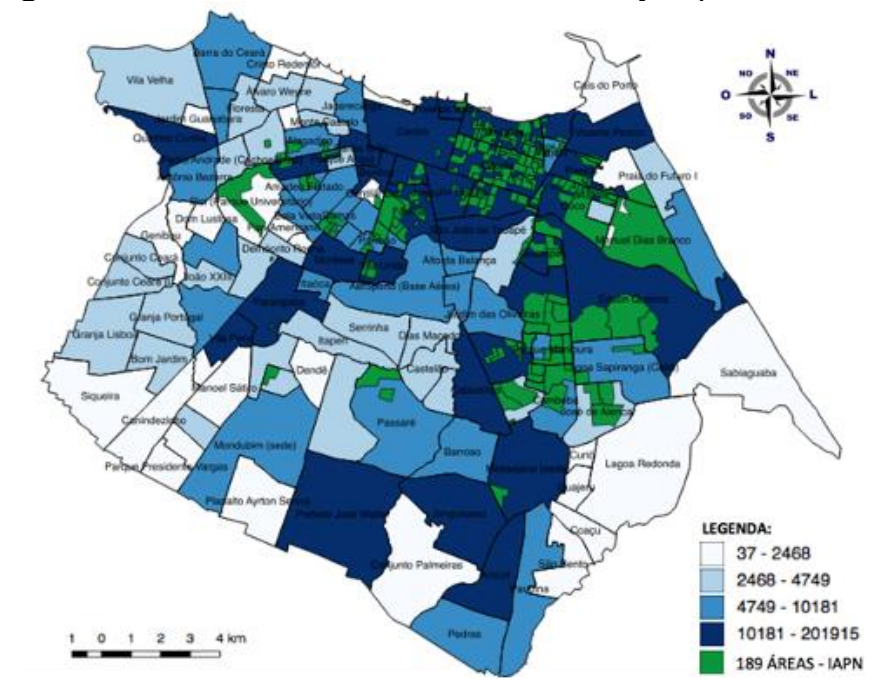

Fonte: Elaboração do autor

As áreas em cor azul são os bairros de Fortaleza com maior metragem de edificações de serviços, dado do observatório da Prefeitura de Fortaleza para 2015. Nesses bairros com maiores empreendimentos de serviços, observa-se que as áreas potenciais de negócio espelhados pelo ILOE, principalmente para as áreas mais bem classificadas, representadas pela cor azul escura, estão instaladas espacialmente exatamente nesses bairros.

\section{CONCLUSÕES}

Criar um índice que traduza uma área potencial de cliente não é tarefa fácil, muitas variáveis podem entrar no contexto. Em um projeto de implantação de empresas, quase todas as variáveis, definidas a partir de premissas a priori, são quantitativas, ou seja, são quase certas. No entanto, uma variável é probabilística, é incerta, que é a demanda efetiva.

Dá para definir ex-ante, quanto será o gasto de energia, mas não dá para afirmar para quantos se irá vender, ou quanto será a demanda efetiva. Essa aleatoriedade pode ser amenizada com premissas científicas fortes. É o que se buscou nesse trabalho com a criação do Índice de Localização Ótima de Empresas - ILOE em um modelo híbrido com análise multicritério, cujo desenvolvimento 
utilizou variáveis socioeconômicas para mapear as áreas censitárias (em torno de $0,4 \mathrm{~km}^{2}$ por área) com maior potencial de negócio para as empresas de médio e pequeno porte da área comercial de varejo.

A partir das áreas definidas pelo ILOE, utilizou-se o método multicritério de otimização para qualificar a tomada de decisão conforme as preferências reveladas pelos decisores. O ILOE definiu espaços potenciais de aproximadamente 0,4 Km2 cada um, mas como estes espaços contêm dezenas de ruas, critérios foram definidos para classificar estas ruas delimitas pelo ILOE com base no MAUT.

Após os filtros, de 756 áreas conforme na Figura 3, chegou-se a 189 áreas de grande potencial de negócio, conforme Figura 4. Salientando-se que as classes sociais " $C$ " e " $D$ " encontram-se dispersas em toda Fortaleza, mas com maior concentração nessa área. Conclui-se que as seguintes premissas sejam priorizadas na escolha da área:

i. Sejam de maior ILOE, indo das 756(Figura 3) áreas para as 189(Figura 4) áreas prioritárias;

ii. Dentro das áreas prioritárias, seja levado em consideração a concentração de serviços e comércio, conforme Figuras 7 e 8;

iii. O ponto(vetor) ótimo $\overrightarrow{z_{3}}=(2,2)$ deverá ser escolhido, significando que a rua(alternativa) com esses atributos é a melhor localização dentro do espaço delimitado pelo ILOE (189), dadas as preferências do empresário(decisor), resultado da otimização multicritério.

\section{REFERÊNCIAS}

AKPINAR, M.; CAN, Ö.; MERMERCIOGLU, M. Assessing the sources of competitiveness of US States. Competitiveness Review, v. 27, n. 2, 2017. http://www.emeraldinsight.com/toc/cr/27/2. https://doi.org/10.1108/CR-02-2016-0014

ALMEIDA, A. T. Processo de Decisão nas Organizações: construindo modelos de decisão multicritério. São Paulo: Editora Atlas. 2013.

ALMEIDA, F. R. S. L. Análise de Localização no contexto de redes varejistas: levantamento das principais técnicas e práticas. Revista de Administração, v.32, n.2, p.36-46, abr./jun., 1997. 
AMIN, S. H., BAKI, F. A facility location model for global closed-loop supply chain network design, Applied Mathematical Modelling, 41, 316-330, 2017.

https://doi.org/10.1016/j.apm.2016.08.030

ARAGÃO, P. S. S. Geomarketing: modelos e sistemas, com aplicações em telefonia. 2004, 94f. Dissertação (mestrado) - Instituto de Computação da Universidade Estadual de Campinas. Campinas: UNICAMP, 2004.

BARROS, M. D.; COSTA, H. G.; SILVA, G. B.; BARCELOS, M. R. S. Análise Multicritério em Dados sobre Empreendedorismo: Um Estudo Bibliométrico. Revista Produção Online, v.17, n. 3, p. 1069-1089, 2017. https://doi.org/10.14488/1676-1901.v17i3.2764

BAVIERA-PUIG, A.; BUITRAGO-VERA, J.; ESCRIBA-PEREZ, C. Geomarketing models in supermarket location strategies. Journal of Business Economics and Management, v. 17, n. 6 , p. 1205-1221, 2016. https://doi.org/10.3846/16111699.2015.1113198

BRIOZO, R. A., MUSETTI, M. A. Método multicritério de tomada de decisão: aplicação ao caso da localização espacial de uma Unidade de Pronto Atendimento - UPA 24 h. Gest.

Prod., v. 22, n. 4, p. 805-819, 2015. https://doi.org/10.1590/0104-530X975-13

BRITO, I. E. W. J.; SPEJORIM, W. Gestão estratégica de armazenagem. 1. ed. Curitiba: IESDE Brasil S. A, 2012.

CAVION, Renata; PHILIPS, Jürgen. Os Fundamentos do Geomarketing: Cartografia, Geografia e Marketing. COBRAC 2006 - Congresso Brasileiro de Cadastro Técnico Multifinalitário - UFSC Florianópolis · 15 a 19 de Outubro, 2006.

CHACÓN-GARCÍA, J. Geomarketing techniques to locate retail companies in regulated markets. Australasian Marketing Journal (AMJ), v. 25, n. 3, p. 185-193, 2017. https://doi.org/10.1016/j.ausmj.2017.06.001

CHEN, Y., KILGOUR, D.M., \& HIPEL, K.W. (2008). Screening in multiple criteria decision analysis. Decision Support Systems. 45, 278-290. https://doi.org/10.1016/i.dss.2007.12.017

CORRAR, L. J.; PAULO, E.; DIAS, J. M. F. Análise Multivariada. 1. ed. São Paulo: Atlas, 2014.

DE LA VEGA, D.S., VIEIRA, J. G. V., TOSO, E.A.V., \& DE FARIA, R.N. A decision on the truckload and less-than-truckload problem: An approach based on MCDA, International Journal of Production Economics, 2017. https://doi.org/10.1016/j.ijpe.2017.09.013

ENGEL, J. F.; BLACKWELL, R. D.; MINIARD, P. W. Comportamento Do Consumidor. Rio de Janeiro: Livros Técnicos e Científicos Editora S.A., 2000.

EHRLICH, P. J. Modelos Quantitativos de Apoio as Decisões. RAE - Revista de Administração de Empresas, v. 36, n.1, p. 33-41, jan./mar., 1996. https://doi.org/10.1590/S0034-75901996000100006

ESTER, E. D. R.; LOUREIRO, A. S.; LEITE, R. D. Estudo de Localização para um Centro de Distribuição na Região de São Paulo para Operações com Produtos Hortifruti. In: CONGRESSO DE PESQUISA E ENSINO EM TRANSPORTE DA ANPET, 32., 2018. [Anais...]. Gramado, RS, Brasil, 04 a 07 de Novembro, 2018. 
GANESHA, H. R.; AITHAL, P. S.; KIRUBADEVI, P. Ideal Store Locations for Indian Retailers: an empirical study. International Journal of Management, Technology, and Social Sciences (IJMTS), v. 5, n. 1, p. 215-226, 2020. Disponível em: https://ssrn.com/abstract=3611474. https://doi.org/10.47992/IJMTS.2581.6012.0090

GOMES, F. A. M.; GOMES, C. F. S. Princípios e métodos para tomada de decisão: enfoque multicritério. 6. Ed. São Paulo: Atlas. 2019.

HERNÁNDEZ, T.; BENNISON, D.; CORNELIUS, S. The organizational context of retail location decision making. GeoJournal, v.45, n. 4, p.295-308, 1998. https://doi.org/10.1023/A:1006989131869

JANNUZZI, P. M.; MIRANDA, W. L.; SILVA, D. S. G. Análise multicritério e tomada de decisão em políticas públicas: aspectos metodológicos, aplicativo operacional e aplicações.

Informática Pública, v.1, n. 11. 2009.

KITA, P.: SZCZYRBA, Z.; FIEDOR, D.; LETAL, A. Recognition of business risks when purchasing goods on the Internet using GIS: experience from Slovakia. Electronic Commerce Research, v. 18, n. 3, p. 1-17, 2017. https://doi.org/10.1007/s10660-017-9276-5

MANGINI, E. R.; ROSSINI, F. H. B.; SANTOS, A.; URDAN, A. T. Análise de Localização de Estações Ferroviárias e Uso de Geomarketing. G\&DR, v. 13, n. 2, p. 129-152, mai/ago. 2017.

MATTEI, T. F.; MATTEI, T. S. Métodos de Análise Regional: um estudo de localização e especialização para a Região Sul do Brasil. Revista Paranaense de Desenvolvimento, v.38, n.133, p.227-243, jul./dez. 2017.

MELO, T. E. Uso e Aplicação de modelos econômicos em sistemas de informação para geomarketing. 2003, 71f. Dissertação (mestrado) - Instituto de Computação da Universidade Estadual de Campinas. Campinas: UNICAMP, 2003.

NUNES, N. F.; SILVA, A. V. Modelos, Métodos e Teorias Utilizados no Estudo da Decisão e suas Aplicações. Humanidades \& Tecnologia em Revista. v.16, Ano XIII, jan./dez, 2019.

PARENTE, J.; KATO, H, T. Área de Influência: um estudo no varejo de supermercados.

Revista de Administração de Empresas, v. 41, n.2, p. 46 - 53, 2001. https://doi.org/10.1590/S0034-75902001000200005

PEREIRA, J. A. M.; LIMA, J. F.; STAMM, C. Um estudo da teoria da localização e das políticas públicas: algumas avidências do caso da região Oeste do Paraná. In: SEMINÁRIO INTERNACIONAL DE DESENVOLVIMENTO REGIONAL: Processos, Políticas e Transformações Territoriais, 9., 2019. [Anais...] Santa Cruz do Sul, RS, Brasil, 11 a 13 de set. 2019.

RHIM, H.; HO, T.H.; KARMARKAR, U.S. Competitive location, production, and market selection. European Journal of Operational Research, v.149, p.211-228, 2003. https://doi.org/10.1016/S0377-2217(02)00445-9

SAKR, M.; DALLABRIDA, V. R. O Marketing Territorial Como Estratégia De Construção E Divulgação Da Imagem Dos Territórios: a Indicação Geográfica Como Referência. Revista Brasileira de Estudos Urbanos e Regionais, 2017. https://doi.org/10.22296/2317$\underline{1529.2017 v 19 n 1 p 89}$ 
SANTOS, M. O espaço dividido: os dois circuitos da economia urba- na nos países subdesenvolvidos. 2 ed. São Paulo: EDUSP. 2004.

SILVA, H.; PARENTE, J. G.; KATO, H. T. Segmentação de Baixa Renda Baseada no Orçamento Familiar. Faces (FACE/FUMEC), v. 8, p. 98-114. 2009.

SILVA, S. C. B. M. Centralidade urbana no estado da Bahia: um estudo comparativo. Revista de Geografia, v. 14, n. 2, out. 1987.

SILVA, S. C.B. M. Teoria de localização e de desenvolvimento regional. Revista Geografia, v. 1, n. 2. Rio Claro: Unesp, out. 1976.

SOARES, G. R. L., SOBRAL, M. F. F., \& MORAES, R. A. F. Uso da modelagem multicritério para apoio ao decisor na classificação de riscos de empresas terceirizadas. Revista

Produção Online, v. 20, n. 1, p. 296-315. 2020. https://doi.org/10.14488/1676-1901.v20i1.3612

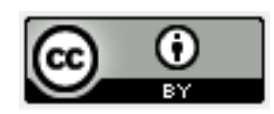

Artigo recebido em: 06/01/2021 e aceito para publicação em: 05/03/2021

DOI: http://dx.doi.org/10.14488/1676-1901.v21i1.4202 